\title{
HEAT TRANSFER ENHANCEMENT USING TWO ISOTHERMAL BLOCKS IN A CYLINDRICAL HEAT EXCHANGER
}

\author{
S. TOUZANI ${ }^{1}$, A. CHEDDADI ${ }^{1}$, M. T. OUAZZANI ${ }^{1}$ \\ 1. Research Team "Energy systems, Mechanical structures and Materials, and Industrial Processes modeling", \\ MOSEM2PI, Mohammadia School of Engineers, Mohammed V University in Rabat. \\ sara.touz@gmail.com, cheddadi.ak@gmail.com, ouazzani@emi.ac.ma
}

\begin{abstract}
The influence of two isothermal blocks placed in a low position $\varphi_{\mathrm{m}}=0.18 \pi$ in a cylindrical heat exchanger is numerically studied. The governing equations are discretized by the centered finite difference method using the ADI scheme. By varying Rayleigh number range between 1000 and 10000, we are interested in the local Nusselt number and the local efficiency variations as a function of the angular coordinate $\varphi$. In one hand, we found that heat transfer is important in the superior zone of the annulus for high $R a$ and in the central zone for low $R a$. On the other hand, compared to the without blocks configuration, heat transfer in the inferior and central zones is improved for all $R a$.
\end{abstract}

Key words: Heat exchanger, isothermal blocks, efficiency.

\section{Introduction}

The addition of fins is considered as one of the frequent methods used to enhance heat transfer inside cylindrical heat exchangers. Nagarani et al [1] described it as an economical and efficient passive method. However, this addition needs to be optimized. Thus, several researchers have been interested in studying fins number, geometry and other parameters in order to obtain a maximum or a minimum heat transfer rate as needed. Rahnama and Farhadi [2] investigated the impact of 2 to 12 radial fins in a cylindrical annulus and concluded that increasing the fins number reduces heat transfer. Other authors studied different fin profiles, such as Iqbal et al [3], who searched for optimal configurations of parabolic fins to obtain maximum convection in two concentric cylindrical pipes. Touzani et al [4] studied natural convection improvement by the use of two isothermal blocks placed in the lower part of a cylindrical annulus. In the same way, we are interested in this work to determine the block contribution on heat transfer increase as well as the impact of the established flow pattern. A comparison with the without blocks case is also presented.

\section{Problem statement}

Figure 1 illustrates the configuration studied. It is composed of a cold outer cylinder at a temperature $T^{\prime}{ }_{o}$ and a hot inner cylinder at a temperature $T^{\prime}{ }_{i}$. These horizontal coaxial cylinders are characterized by a radii ratio $R=r_{o}^{\prime} / r_{i}^{\prime}$ fixed at 2 . The problem is twodimensional. The blocks are isothermal of the same temperature as the inner cylinder and placed on it. They are characterized by: a length $l^{\prime}$, a height $h^{\prime}$ and an angular position $\varphi_{\mathrm{m}}$. The fluid is an incompressible viscous fluid obeying the Boussinesq approximation. The cavity is divided into three zones as a function of the angular coordinate $\varphi$ : inferior for $0 \leq \varphi<\varphi_{\min }$, central for $\varphi_{\min } \leq \varphi \leq \varphi_{\max }$ and superior for $\varphi_{\max }<\varphi \leq \pi$.

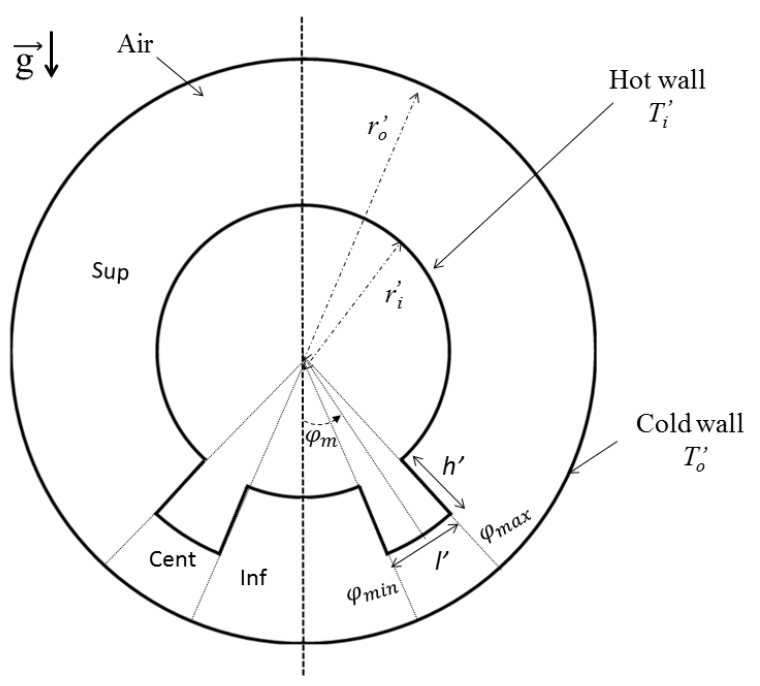

Figure 1: Problem configuration

\section{Mathematical model}

The stream function-vorticity formulation is adopted. The governing equations for two-dimensional flow are written in dimensionless form, as the following (1-8):

$\Delta \psi+\omega=0$ 


$$
\begin{gathered}
\frac{\partial \omega}{\partial t}+\frac{1}{r}\left(\frac{\partial \psi \partial \omega}{\partial \varphi \partial r}-\frac{\partial \psi \partial \omega}{\partial r \partial \varphi}\right)=\operatorname{Pr} \Delta \omega+\operatorname{RaPr}\left(\frac{\cos \varphi}{r} \frac{\partial T}{\partial \varphi}\right. \\
\left.+\sin \varphi \frac{\partial T}{\partial r}\right) \\
\frac{\partial T}{\partial t}+\frac{1}{r}\left(\frac{\partial \psi \partial T}{\partial \varphi \partial r}-\frac{\partial \psi \partial T}{\partial r \partial \varphi}\right)=\Delta T
\end{gathered}
$$

Boundary conditions:

On the inner cylinder $(r=1)$ :

$$
\psi=0, \frac{\partial \psi}{\partial r}=0, \frac{\partial^{2} \psi}{\partial r^{2}}+\omega=0, T=1 \quad \forall \varphi
$$

On the blocks surfaces:

$$
\begin{gathered}
-r=h+1 \text { and } \varphi_{\min } \leq \varphi \leq \varphi_{\max }: \\
\psi=0, \frac{\partial \psi}{\partial r}=0, \frac{\partial^{2} \psi}{\partial r^{2}}+\omega=0, T=1 \\
-\varphi=\varphi_{\max } \text { and } \varphi=\varphi_{\min }, 1 \leq r \leq h+1: \\
\psi=0, \frac{\partial \psi}{\partial \varphi}=0, \frac{1}{r^{2}} \frac{\partial^{2} \psi}{\partial \varphi^{2}}+\omega=0, T=1
\end{gathered}
$$

On the outer cylinder $(r=R)$ :

$$
\psi=0, \frac{\partial \psi}{\partial r}=0, \frac{\partial^{2} \psi}{\partial r^{2}}+\omega=0, T=0 \forall \varphi
$$

Symmetry condition:

$$
\varphi=0, \varphi=\pi: \psi=0, \omega=0, \frac{\partial T}{\partial \varphi}=0 \quad \forall r
$$

The system of equations with boundary conditions (1-8) is numerically solved by centered finite difference using the ADI scheme (Alternating Direction Implicit). In a preliminary work, it has been shown that the mesh $65 \mathrm{x}$ 65 can be considered sufficient to describe the steady state regimes to which we are interested. In this investigation, the same mesh number is considered.

\section{Results and discussion}

The results are presented for a block, of a height $h=0.5$ and a length $l=0.109$, placed in a low position $\varphi_{\mathrm{m}}=0.18 \pi$ and for Rayleigh numbers range varying between 1000 and 10000. Due to the symmetry condition, only the half of the configuration is considered.

The observed flow pattern is characterized, regardless of $R a$, by the presence of two cells: a main and a counterrotating one. The main cell occupies the majority of the annular medium while the counter-rotating cell is found in the inferior zone. This type of bicellular regime is plotted in Figure 2. Isotherms deform and distort with the increase of Rayleigh number.
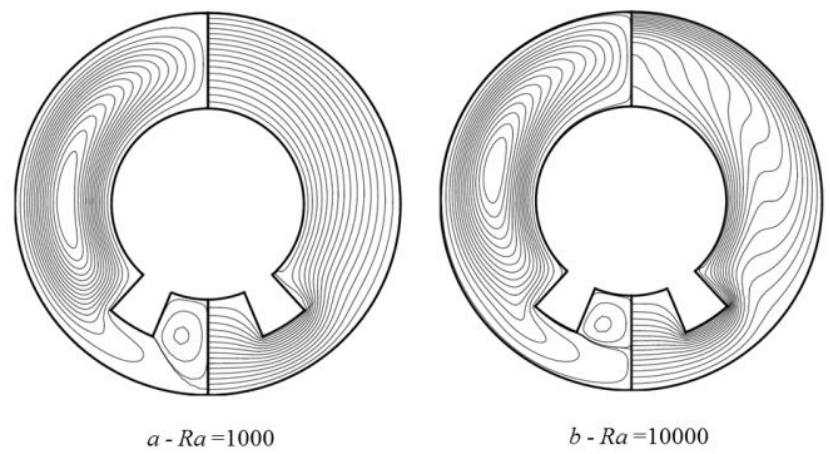

Figure 2: Streamlines and isotherms. $\mathrm{a}: R a=1000, \mathrm{~b}: R a=10000$.

In order to evaluate the isothermal block influence on heat transfer, we are interested in calculating the local Nusselt number expressed by the formula: $N u=-\left.R \ln R \frac{\partial T}{\partial r}\right|_{r=R}$.

Figure 3 gives the variation of $N u$ as a function of $\varphi$ for different $\mathrm{Ra}$. We notice that the curves of $\mathrm{Nu}$ remain ordered as $\mathrm{Nu}$ increases when $R a$ decreases for $0 \leq \varphi \leq 0.23 \pi$ while this order changes for $0.40 \pi \leq \varphi \leq \pi$. For $\quad 0.23 \pi<\varphi<0.40 \pi, \quad$ crossings between the curves take place for all $R a$.

The analysis by zones indicates that in the inferior zone, the counter-rotating cell contributes positively in heat transfer amelioration in this area where $N u$ is varying between 1.19 at $R a=10000$ and 1.47 at $R a=1000$. On the central zone, a maximum of $\mathrm{Nu}$ is observed showing the block heating effect in that zone. In fact, this extremum is the absolute maximum for $R a \leq 2000$. Beyond that maximum position, $N u$ decreases then increases and reaches an absolute maximum value at $\varphi=\pi$ for $R a \geq 3000$. Therefore, it can be concluded that heating caused by the block strongly influences heat transfer increase for $R a \leq 2000$. Beyond this value, the influence of the block, which remains present, becomes less important than the contribution of the main cell close to the summit of the annulus. 


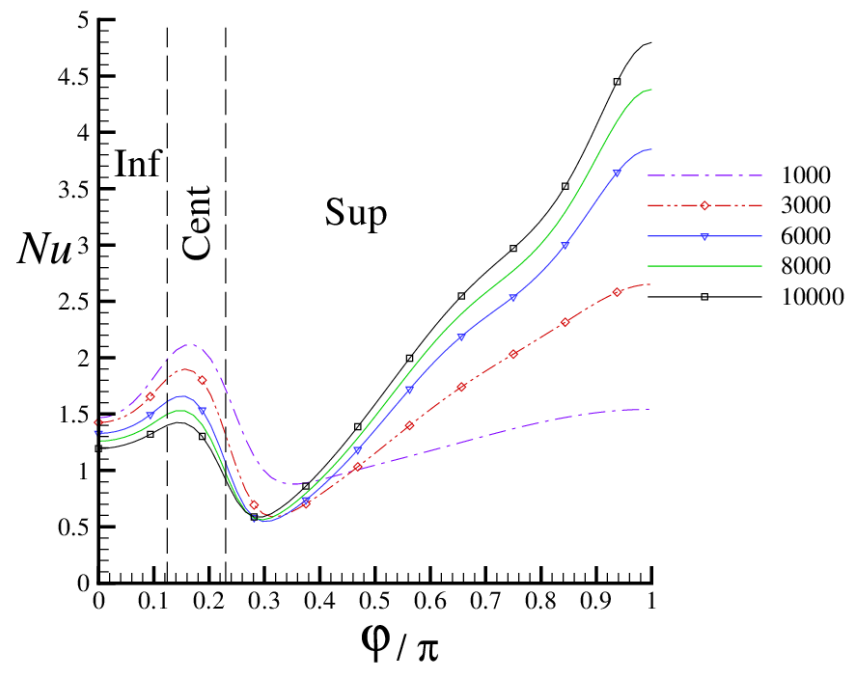

Figure 3: Local Nusselt number variation as a function of $\varphi$ for different $R a$.

To analyze the improvement of heat transfer, we compare the studied configuration to the one without blocks by calculating the local efficiency.

The relative difference between $\mathrm{Nu}$ and the local Nusselt number, $N u^{0}$, of the configuration without blocks, representing local thermal efficiency as a function of $\varphi$,

is defined by : $\eta_{l}=\frac{N u-N u^{0}}{N u^{0}}$.

For low Rayleigh numbers, $\eta_{l}$ increases with $\varphi$ augmentation to $210.47 \%$ for $R a=1000$ and to $467.61 \%$ for $R a=5000$ (figure 4). It drops to near-zero values beyond $\varphi=\varphi_{\max }$ for all Rayleigh numbers.

As observed for $\mathrm{Nu}$, the counter-rotating cell effect on heat transfer increase is small compared to the heating induced by the block for $R a<6000$. A maximum of $\eta_{l}$ is observed at a position $(\varphi=0.16 \pi)$ slightly equal to $\varphi=\varphi_{\mathrm{m}}$. For $R a \geq 6000, \eta_{l}$ maximum is observed at $\varphi=0$ with a value of $610.18 \%$ for $R a=10000$. This shows that the fluid movement caused by the counterrotating cell and the heating induced by the block, complement each other and lead to heat transfer enhancement. Hence, we can say that only thermal transfer in the lower and central zones is improved.

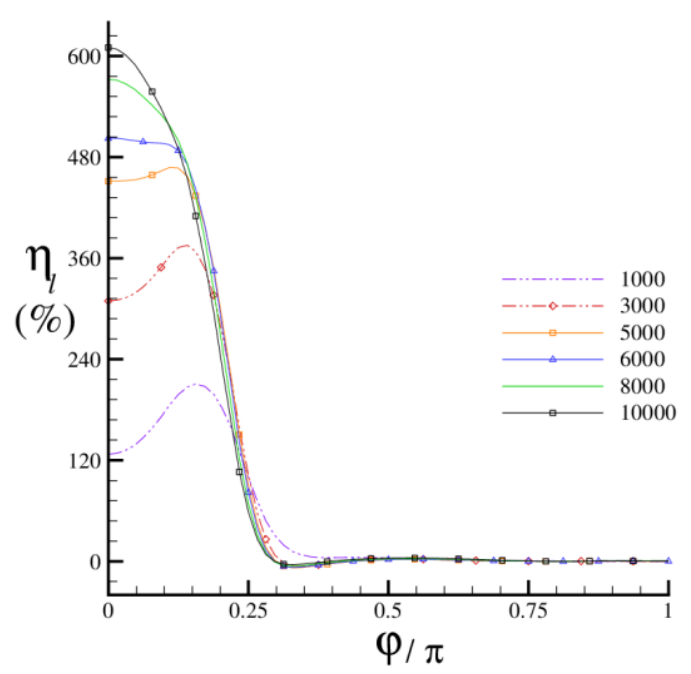

Figure 4: Local efficiency as a function of $\varphi$ for different $R a$.

\section{Conclusion}

The present work is a numerical study to determine the contribution of two isothermal blocks, placed in a low position, to the improvement of heat transfer within a cylindrical heat exchanger. It is found that the superior zone of the annulus offers an important heat transfer for high $R a$ while it is the central zone, where the block is placed, which plays that role for low Rayleigh numbers. Compared to the without blocks configuration, regardless of $R a$, the bicellular regime found influences positively the heat transfer within the configuration by improving it at the inferior and central zones.

\section{References}

[1] N. Nagarani, K. Mayilsamy, A. Murugesan and G. Sathesh Kumar, Review of utilization of extended surfaces in heat transfer problems, Renewable and Sustainable Energy Reviews, 29 (2014) 604-613.

[2] M. Rahnama, M. Farhadi, Effect of radial fins on two-dimensional turbulent natural convection in a horizontal annulus, International Journal of Thermal Sciences, 43 (2004) 255-264.

[3] Z. Iqbal, K. S. Syed, M. Ishaq, Optimal convective heat transfer in double pipe with parabolic fins, International Journal of Heat and Mass Transfer, 54 (2011) 5415-5426.

[4] S. Touzani, A. Cheddadi, M. T. Ouazzani, Numerical study of laminar natural convection in a finned annulus: low isothermal blocks positions, Journal of Engineering Physics and Thermophysics, (2019) (accepted). 